\title{
“I Am Strong. Mentally Strong!": Psychosocial Strengths of International Graduate Students of Color
}

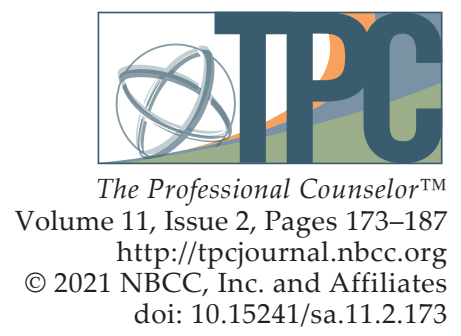

\author{
S Anandavalli, L. DiAnne Borders, Lori E. Kniffin
}

Positioned at a unique intersection of managing academic pressures and embodying racial and ethnic minority identity status, international graduate students of color (IGSCs) are frequent targets of multiple stressors. Unfortunately, extant counseling literature offers counselors little information on the psychosocial strengths IGSCs employ (e.g., strong familial bond, friendships) to cope with such stressors. To address this gap, interviews with eight IGSC participants were conducted and analyzed using interpretive phenomenological analysis and the lens of the intersectionality framework. Five psychosocial strengths were identified -familial support, social connections, academic aspirations and persistence, personal growth and resourcefulness, and resistance and critical consciousness. Recommendations for employing an asset-based approach in counseling and counselor education are offered.

Keywords: international graduate students of color, psychosocial strengths, racial and ethnic minority, intersectionality, interpretive phenomenological analysis

Over 1 million international students were enrolled in higher education programs in the United States in 2020 (National Association of Foreign Student Advisers [NAFSA], 2020). Thousands of them are confronted with varied stressors, including linguistic barriers (Mori, 2000), isolation (Sato \& Hodge, 2015), and xenophobia (Pottie-Sherman, 2018). The recent COVID-19 pandemic has added to their mounting stress, pushing them toward symptoms of severe mental illness, including chronic fear and anxiety (Chirikov \& Soria, 2020). Extant literature offers strong evidence that international graduate students of color (IGSCs) are particularly vulnerable to stressors because of their academic responsibilities and experiences in the racialized society of the United States (George Mwangi et al., 2019; Lee \& Rice, 2007; Lemieux et al., 2020).

To support IGSCs as they confront multiple challenges, scholars have been called to examine this population's psychosocial strengths (Pendse \& Inman, 2017; Straker, 2016), as strengths-based counseling empowers and builds resilience among members of marginalized communities (Craig, 2013; Day-Vines \& Terriquez, 2008; Toporek \& Cohen, 2017). Regrettably, a thorough literature review yielded no peer-reviewed counseling articles that specifically addressed the strengths of IGSCs. The only related counseling study was by Yakunina and colleagues (2013), who offered insight into the strengths of international students. However, they failed to examine the specific strengths of IGSCs and gathered data only on the overall international student experience.

Thus, to explore the psychosocial strengths of IGSCs, we used interpretive phenomenological analysis (IPA; Smith, 1996) and adopted the lens of the intersectionality framework (Crenshaw, 1989). We review the literature addressing IGSCs' psychosocial strengths and offer results professional counselors can use in an asset-based approach with this community.

S Anandavalli, PhD, NCC, LPC (Intern), is an assistant professor at Southern Oregon University. L. DiAnne Borders, PhD, NCC, ACS, LPC, is a professor at the University of North Carolina at Greensboro. Lori E. Kniffin, PhD, is an assistant professor at Fort Hays State University. Correspondence may be addressed to S Anandavalli, Southern Oregon University, 1250 Siskiyou Blvd, Ashland, 0R 97520, anandavas@sou.edu. 


\section{Literature Review}

\section{Intersectionality}

Using the intersectionality framework, Crenshaw (1989) noted that racial, linguistic, class, and other identities intersect to inform an individual's position of privilege and marginalization. These intersecting identities result in a heterogeneity of experiences within the international student community. The concept of intersectionality highlights the differing and inequitable influences of systemic oppression on diverse groups. In other words, the experiences of international students are dictated by their identities of race, ethnicity, level of education, language, and gender.

International students of color, because of their racialized ethnic identities, often bear the brunt of discrimination and toxic hate crimes (Chirikov \& Soria, 2020; Dovchin, 2020; George Mwangi et al., 2019; Lee \& Rice, 2007). Lee and Rice (2007) noted that international students of color in their study were often targets of racial slurs, violence, verbal insults, and discrimination. The internalized effects of colonialism and colorism were evident in how teachers and domestic students responded to African international students, including questioning their intelligence and legitimacy to be in graduate-level classrooms (George Mwangi et al., 2019). Students disclosed that they frequently felt "crazy" because of microaggressions by unrelenting domestic students in their classrooms (George Mwangi et al., 2019). Recently, Yao (2018) found that Chinese international students experienced both overt and implicit discrimination as a result of their nationality, language, and cultural background, having significant mental health impact. Thus, it is evident that the experiences of international students of color are significantly different from that of their White counterparts (Yao et al., 2020).

Additionally, with their demanding academic and research expectations, international graduate students (IGSs) constitute a particularly unique community (Girmay, 2019; Lin \& Scherz, 2014). Lemieux and colleagues (2020) noted that the recent directive by U.S. Immigration and Customs Enforcement (ICE), which ordered international students to leave the United States if their fall 2020 courses were entirely online, had a profoundly deleterious impact on IGSs (ICE, 2020). IGSs usually serve as teaching assistants and research assistants, and they are often part of large-scale research and development projects. Lemieux and colleagues argued that despite bringing significant technological, cultural, and economic advantages to the United States, the order devalued the contributions of IGSs amidst the global health pandemic of COVID-19. Furthermore, the excessive pressure to engage in research and publications often had a detrimental impact on international students. Participants in a recent study by Click (2017) discussed that navigating a new academic system and responding to professional expectations were challenging and moved at least one participant in the study to tears. Thus, IGSs are confronted with multiple challenges that require urgent attention.

\section{Psychosocial Strengths}

Scholars have noted that practitioners' knowledge and utilization of marginalized clients' (e.g., Latinos, African Americans, LGBT individuals) psychosocial strengths often results in increased resilience, leadership, hardiness, and self-efficacy (Craig, 2013; Day-Vines \& Terriquez, 2008). However, despite the efficacy of strengths-based counseling with minorities, the strengths of marginalized groups are often unknown to or underutilized by many professional counselors (Anandavalli, 2021; Craig \& Furman, 2018; Harry et al., 2005; Tomlinson-Clarke \& Georges, 2014). Given the extensive challenges the IGSC community faces, a strengths-based approach to counseling might prove to be especially beneficial. Regrettably, a thorough literature review on Google Scholar, PsycINFO, and SocINDEX using over nine search terms, including international student mental health, international students, strengths-based, and international students assets, indicated that no strengths-based counseling articles were available that addressed the experiences of IGSCs. 
Given the paucity of counseling research on IGSCs' psychosocial strengths, this section includes inquiries from non-counseling studies and also those that address the larger international student community. A review of the literature suggested there are two broad themes of psychological strengths-interpersonal strengths and intrapersonal strengths.

\section{Interpersonal Strengths}

In the context of academic advising, He and Hutson (2018) found that Chinese international students in their study relied on their families' support to overcome adversities and persist in their academic programs. Families' significant efforts (e.g., financial and emotional support) to send their children abroad fueled the participants to make the most of their education in the United States (He \& Hutson, 2018). Similarly, within the field of psychology, Moores and Popadiuk (2011) observed that as international students in the study navigated through stress in the host country, their families' support was a reminder of their cultural identity.

In addition to families, international students' friendships are potentially another asset. Given that they experience multiple sociopolitical stressors (Anandavalli et al., 2020), these students rely on each other for support (e.g., Wang \& Hannes, 2014). Adopting an international relations perspective, Wang and Hannes (2014) noted their participants made plans to cook meals together and invited one another to cultural events to avoid the effects of isolation in a foreign country. The findings were used to inform faculty and policy makers in the Flemish community.

\section{Intrapersonal Strengths}

Scholars have also identified international students' intrapersonal strengths, including hardiness, personal growth initiative (Yakunina et al., 2013), and a deep desire to succeed (Wick et al., 2019). Yakunina et al. (2013) gathered data from over 300 international students in the United States to test if personal growth initiative (drive to improve oneself), hardiness (resilience), and universal-diverse orientation (ability to appreciate cultural similarities and differences) predicted the students' acculturative stress and adjustment in the United States. Their sample included international students from diverse ethnic, educational, and linguistic backgrounds. The researchers found that participants' personal growth initiative predicted their adjustment in the United States. Relatedly, Yakunina and colleagues also found that hardiness had a positive effect on adjustment and an underlying impact on participants' levels of acculturative stress. Furthermore, international students' universal-diverse orientation determined the extent of their adjustment. Thus, participants who reported greater universal-diverse orientation tended to experience reduced acculturative stress, which in turn predicted better adjustment.

Adopting an interdisciplinary and critical perspective to studying abroad, Wick and colleagues (2019) found that undergraduate Latinx students from the United States leveraged multiple strengths during their study abroad experience. In this study, Wick and colleagues employed Yosso's (2005) community cultural wealth theory to identify participants' potential strengths. Wick and colleagues noted that the participants effectively used their familiarity with Latinx cultures, Spanish language, and a deep desire to succeed in helping them make the most of their study abroad experiences. Wick and colleagues also noted that the international students experienced personal growth and reported additional strengths such as a heightened sense of social responsibility and critical consciousness, or a deeper understanding of how oppression and marginalization impact lived experiences.

\section{Purpose of the Present Study}

Although knowledge of international students' psychosocial strengths has been documented in related fields, data on the same in counseling is limited. Consequently, counselors are ill-equipped to utilize 
these in the clinical setting, and clients may not be able to reap the benefits of strengths-based counseling (e.g., increased resilience, self-efficacy). Given the evidence in favor of strengths-based counseling for minoritized communities, the purpose of this study was to offer a strengths-based perspective of the coping strategies used by IGSCs. Our goal was to capture the psychosocial strengths IGSCs employ to support their well-being. Thus, our research question was, "What are the psychosocial strengths that IGSCs employ to support their well-being during their study abroad experience in the United States?"

\section{Method}

The present inquiry was the first part of a larger study. In this first part of the study, we focused on the strengths of IGSCs. We applied IPA (Smith, 1996) to explore IGSCs' use of psychosocial strengths to support their well-being. IPA is an approach that allows researchers to maintain the uniqueness of each participant's subjective lived experiences while laying emphasis on the contextual nature of interpretation (Smith, 1996). In other words, people's interpretation of their lived experiences is grounded in the specific social and cultural context of their narratives. The primary focus of IPA is to examine how individuals make sense of their experiences and minimize any chance of researchers imposing their personal beliefs and expectations on the participants' experiences (Smith, 1996). All research activities were in compliance with the National Board for Certified Counselors (NBCC) Code of Ethics (NBCC, 2016).

\section{Researcher Roles and Trustworthiness}

There are four aspects of effective qualitative research (Guba, 1981), and each aspect was pursued by the team through varied ways: credibility (e.g., member checking, two coders), transferability (e.g., multiple data points for each subtheme), dependability (e.g., utilizing external auditor's feedback), and confirmability (e.g., field notes). The research team for this study was composed of three cisgender women. At the time of the study, the first author, Anandavalli, identified as an IGSC from India. The second author and auditor for the study, Borders, is a White American counselor educator and has worked with IGSCs from multiple countries. The third author, Kniffin, also a White American, has experiences working with international students in her previous professional roles. Each researcher's ongoing reflexivity journal, an integral part of IPA, served to identify potential blind spots specifically centering around stereotypes and perceptions surrounding the IGSC community, and the role of institutions and structures in impacting IGSCs' mental health.

To establish meaningful trustworthiness, Anandavalli immersed herself in the IGSC community at a medium-sized university in the United States for about 2 years before beginning the study. The cultural immersion and community-building opportunity allowed for the research question(s) for the larger study to emerge from collective reflections with the participating community. Individuals with prior relationships with the research team were not selected as participants to ensure an ethical inquiry and avoid undue influence of coercion and/or social desirability.

\section{Participants}

Based on Smith and Osborn's (2007) recommendation, a sample size of seven to eight participants was deemed sufficient for the current study. In this IRB-approved study, the inclusion criteria were that participants needed to: (a) be at least 18 years of age, (b) be proficient in English (interview language), (c) hold a valid F1 or J1 visa (higher education visas in the United States), and (d) be enrolled in any graduate program in the United States. They were also required to self-identify as a person of color and should have lived in the United States for not longer than 2 years, given the rapid effects of acculturation to dominant culture and resulting changes in their mental health experiences (Erichsen \& Bolliger, 2011). 
Recruitment flyers included details on the purpose of the study and contact information for the first author. The flyers were shared via social media and through communication with various international students' offices at regional universities and colleges. Using convenience and snowball sampling, a total of 23 participants responded to the recruitment announcement. However, 12 did not meet inclusion criteria and three did not respond until months after the completion of data analysis (because of time constraints on the interviewees' part). Eight were eligible and reviewed the IRB-approved consent form before completing the individual, semi-structured interview. Participants were invited to choose a name they preferred for the study. Participants were from India (Cheryl, Jay, and Bansal), China (K. S. and T. L), the United Kingdom (M. F.), Brazil (A. Z.), and Indonesia (Fani). On completion of the interview, each participant received a $\$ 10$ gift card. All participants in the present study identified as female, although no explicit screening for gender identity was imposed by the researchers.

\section{Data Collection}

Before the audio-recorded interview, each potential participant completed a consent form and an online demographic questionnaire to determine their eligibility. Using the interview schedule, Anandavalli interviewed eligible participants. The open-ended, semi-structured interview had five primary questions: 1) Who and what supported you in adjusting to American culture, especially with your education and social life [e.g., ethnic groups]? 2) What has been your experience supporting other international students during their transition period? 3) How have you changed because of the study abroad experience? 4) What were some resources [within campus and community], if any, that you used to maintain your academic success? and 5) What were some resources [within campus and community] that you used to take care of your mental health? Appropriate follow-up questions were employed based on individual participants' responses (e.g., "Tell me more about what you mean when you say your faith held you together during the challenging times"). Three interviews were conducted via Zoom and five were completed face-to-face at a location of the participant's choice, ranging from 60-120 minutes in length ( $M=72$ minutes). Anandavalli maintained field notes on participants' presentation during the interview (e.g., body language, tears) to gain deeper understanding of their narratives.

\section{Data Analysis}

Anandavalli also transcribed all interview recordings. The analysis team was composed of two coders, Anandavalli and Kniffin, and an auditor, Borders. All have had training in IPA methodology and/or coding through graduate-level coursework and prior experience in conducting similar inquiries. Adhering to Smith and Osborn's (2007) recommendations, we followed an open-coding protocol. Each interview transcript was a unit of analysis. Anandavalli and Kniffin read the first transcript multiple times to become familiar with the material. Next, similarities and differences within a participant's narrative of psychosocial strengths were noted, and similar experiences were merged under a common emergent theme. Similar themes within one participant's narrative were then clustered together to construct superordinate themes. The goal was to reduce the volume of the detail in a participant's narrative while also maintaining the uniqueness of their experience. Then, through consensus, Anandavalli and Kniffin developed a shared list of superordinate themes for the first participant's transcript. The same procedure was followed for the remaining seven participants. Borders' feedback was incorporated at various stages. After coding all the individual interviews, Anandavalli and Kniffin created a broad theme list based on each participant's superordinate themes. The broad theme list reflected the overarching experiences of the eight IGSCs. Corresponding individual and broad themes were shared with each participant to receive relevant feedback (member checking) with all participants responding that the themes were accurately reflective of their experiences. No changes were requested by the participants. 


\section{Results}

Five broad themes, with related subthemes, emerged from the eight interviews with IGSC participants. The five themes of familial support, social connection, academic aspirations and persistence, personal growth and resourcefulness, and resistance and critical consciousness are presented below, supported by participants' quotes that elucidate their relevant experiences. The themes are presented in descending order of frequency in the participants' narratives.

\section{Familial Support}

In this broad theme, all eight participants described the crucial role their family played in helping them remain motivated and succeed as international students in the United States. Their narratives indicated that family support was nuanced and was expressed in varying ways, including offering emotional and financial support. Furthermore, participants also disclosed that their idea of "family" included relatives and community members. Three subthemes were identified under this theme: familial ambition, emotional support from parents, and support from extended family.

Familial ambition, or family members' commitment to help IGSCs succeed during their study abroad experience, despite challenges, was evident in participants' narratives. Parents, cousins, and community members were deeply involved in helping the participant succeed and achieve something "great," as Jay described. She claimed, "My family's very supportive ... they want me to do some great thing ... and for that they support all the time."

Cheryl noted that her parents were dedicated to her professional development and worked hard to ensure she made informed decisions regarding her higher education. She recollected a pivotal moment in her education when she had to decide if she wanted to accept a college admission offer from a university in the United Kingdom:

They always said, "No matter what you study, do your best in it." So, when I had to decide about studying abroad ... my mom, she literally found people in her circle who studied abroad ... for me to connect with. And make the right decision.

Relatedly, a second subtheme of emotional support from parents emerged. Through phone calls and texts, IGSCs in the study sought their family's support when they felt challenged and stressed. For instance, Bansal, an IGSC from India, recognized that her parents' support was crucial to her. Their reassurance and communication of support played an important role in her well-being: "You require so much support from your parents when you are studying abroad.... And they say, 'No matter whatever happens, we're there for you."' Similarly, K. S., a doctoral student, sought her mother's support, especially during moments of acute stress. With intense pressure in her STEM research lab and communication challenges as a non-native English speaker, she admitted that her mother's support was important in the initial months: "I always told my mom, 'I am now under pressure again, please say something to comfort me,' and sometimes that works. She is amazing."

Four participants also referred to how family members, including extended relatives, were invested in their education and offered support in diverse ways. Support from extended family was the third subtheme. They also said that in their native cultures, the separation between immediate and extended family was unusual. For instance, Jay recalled that she wished to join a lab that had sufficient funding and scientific equipment to support her innovative research: "My uncle is an education consultant in 
India ... so he searched the best universities in the U.S. with good scholarships for me ... that was so helpful!" Bansal, a first-generation study abroad student, recounted that she sought support from her cousin who had recently completed his higher education as an international student in the United States. According to her, his perspective and experiences helped her prepare for the cultural and academic transitions involved with studying abroad:

My cousin is also an international student here in the U.S. He gave me a lot of advice, so I was pretty prepared for the ups and downs. He advised, "Be flexible and open." So, his experiences prepared me for the best and worst.

\section{Social Connections}

All participants discussed narratives of supportive and challenging moments as they built social connections in the United States. Their social connections were classified into two subthemes: support from Americans and an international student network.

Within the subtheme of support from Americans, we noted that all participants experienced both positive and challenging interactions with local Americans. Jay, for instance, applied for a local host-family program through her campus initiative and sought their help in the early months. Although it has been over a year since she moved to the United States, her host family has continued to help her in multiple ways. "They showed me several shops and vegetarian restaurants ... they're still helpful to me and they are still treating me as their niece." According to her, she made efforts to maintain her connection with her host family, as they taught her about social and cultural norms in the American South.

Three participants received guidance from their American peers. For example, K. S. articulated that she found a strategy to overcome her limited English proficiency: "I built friendships with my American classmates. They're really nice and helpful. They know . . I can't always understand what the professor is saying. After class, I asked them to send me their notes and they did." However, A. Z. struggled to build a deeper relationship with her American counterparts, who she perceived to be less relational than people in her culture. She found them disinterested in getting to know her and her Brazilian heritage: "I want to make friends with American students ... but Americans like to study on their own ... maybe competition on grades. But Americans in my class usually don't like to talk to me or other immigrants."

Building an international student network was another subtheme. All participants disclosed that they sought and offered extensive help to fellow international students. Fani, a student from Indonesia, shared that, "As an international student, we don't really have family here. So, when I meet other international students, we kind of help each other." Participants also offered practical aid to one another. M. F. employed her past experiences of navigating through a foreign country and offered guidance to younger international students: "This girl, from Jamaica, I invited her home for a home cooked meal. I help her with her big shopping once a month and let her use my car and Costco card. Just yeah ... wanted to be helpful."

T. L. disclosed that she countered feelings of loneliness by developing strong bonds with her Chinese friends, who gave her a familial experience:

We traveled a lot and, like to release your stress, you can hang out with a group of friends. And have some fun because they can get what you mean. ... A sense like you have family here. Then they can give you that feeling. 


\section{Academic Aspirations and Persistence}

Although IGSCs experience multiple stressors, all participants shared they were ambitious and keen on excelling in their education. As they persevered in their education, they experienced a deep sense of pride in their achievement. Experiences under this theme were categorized into three subthemes: academic persistence, pride in achievement, and ambition.

The first subtheme was academic persistence. Participants disclosed that despite adversities, they were determined to excel in their education. M. F., a mother of two adolescent girls, was dedicated to completing her doctoral education: "I came here with two daughters ... and being a doc student, it's hard. But I have learned a lot in my program and grown a lot. I plan to successfully ... complete the program in one to two years." Similarly, for T. L., adjusting to U.S. classrooms meant getting used to the English-dominated, participatory culture in American classrooms: "The language barrier makes you feel like an intelligence barrier. The Americans speak so fast .. . it's not easy. But I didn't give up."

Three individuals also shared a sense of pride in their achievements. Thus, the second subtheme was pride in achievement. A. Z., for instance, shared her feeling of happiness and confidence as she performed well in her dissertation proposal defense: "When I finished my proposal ... it felt so good that I did [it] on my own." Participants also talked about how they viewed their educational journey as being composed of multiple milestones, and with each passing milestone, they experienced a renewed sense of achievement. Bansal explained, "It is a sort of an accomplishment, a feeling that 'Yes, you did this,' and you reach the end of the line finally, this is what you gain."

The third subtheme was ambition. Five participants reported that they were ambitious and sought out the strongest chances of succeeding in their respective careers. Cheryl admitted, "I want to get trained by the best ... I want that stamp of approval ... that I am getting the best training."

\section{Personal Growth and Resourcefulness}

All participants' study abroad experiences were dotted with several moments of growth and personal development. Participants' experiences of overcoming obstacles were grouped into three subthemes, which were self-reliance, self-discovery, and use of campus resources.

Three participants shared that they had come to rely on themselves and gained a deeper sense of confidence and self-reliance as a result of studying abroad. Bansal said that she gained a sense of efficacy and faith in her ability to cope with adverse situations: "Back in India ... I would be afraid." However, she said, "It [being an IGSC] shapes you in a way that now you don't fear that I cannot conquer this. ... if something comes up, I can deal with it. Now, I am strong. Mentally strong!"

Participants recognized that even in the face of adversity, they believed they were capable of overcoming challenges as a result of the study abroad journey. Fani said that she had come to embrace her self-confidence and agency: "If I don't have friends who help me, I can just figure it out by myself."

Four participants also discovered aspects of themselves as a result of studying abroad (self-discovery). Jay shared that her identity was "revealed" as a result of studying in the United States and being away from her family: "When I came to a new place, I needed to start explaining about myself. And I got to understand myself and my culture, and all the strengths I have."

Under the subtheme of use of campus resources, participants, including Cheryl and M. F., noted that they took on the responsibility to ensure they built supportive networks in the community and strong 
relationships with library and department staff. Relatedly, A. Z. reported that she regularly attended Graduate Student Association events and went to the campus gym to ensure she did not feel isolated. She shared, "I realized I had to do something about this. I will go crazy otherwise."

Fani recognized that as a Fulbright scholar she would lose her scholarship if did not meet the strict grade criteria for each term. Although she could not excel in her first midterms as an international student, she overcame that challenge:

All of my midterm exam ... pretty bad score really. I was so stressed. But I met them [professors] during the office hours, and it's really helped me.... I asked him so many questions and went to every single office hour meeting.

\section{Resistance and Critical Consciousness}

Despite being surrounded by dominant American culture and norms, four participants found several ways to resist the mindless adoption of the U.S. way of life. They retained their home cultural practices and shared observations of injustices and biases embedded in the American society. The two subthemes were retaining home culture and critical consciousness.

Under the subtheme of retaining home culture, K. S. shared that despite being away from her home in China, she continued to find ways to celebrate her cultural events: "The other Chinese students and I meet for Chinese New Year ... even though we are away from home ... we will celebrate to bring happiness." Similarly, A. Z. talked about listening to Brazilian podcasts and music as a way to retain her link with her home culture. Participants also fostered resistance through retaining their faith and religious beliefs, which often stemmed from their home cultures. M. F., a Catholic, shared that "faith is always there for me. I do believe God is looking out for me. There is ... someone looking out for me."

When asked what potential injustices they had observed in the host country and how they addressed them, four IGSCs shared that they gained deeper awareness of embedded biases and injustices within the American society. Their narratives were indicative of heightened critical consciousness. Cheryl shared:

I think getting educated about what a microaggression is ... helped me label the subtle discrimination I was exposed to. ... In my first semester, I realized my professors weren't as harsh on me as they were for White students ... almost patronizing. Like I am not good enough to meet their White standards.

Participants also questioned and challenged the status quo as opposed to accepting it. A. Z. recognized that she did not want to subscribe to the larger "workaholic" culture in her research lab. She wanted to maintain her cultural practices of work-life balance with time allocated for self-care. T. L. said, "I did not notice anything wrong here. Not yet." However, she acknowledged that having grown up under a relatively more restrictive government and culture, "everything here [in the United States] felt more free."

\section{Discussion}

For ease in discussion and in alignment with our literature review, we grouped participants' descriptions of various psychosocial strengths as interpersonal strengths (familial support, social connections) or intrapersonal strengths (academic aspirations and persistence, personal growth and resourcefulness, and resistance and critical consciousness). 


\section{Interpersonal Psychosocial Strengths}

IGSCs in the study relied on an intricate network of relationships to support their well-being. These networks included family, relatives, and other international students. Participants' narratives indicated that their families played a significant role in actively guiding and supporting them to further their professional development. Furthermore, the pervasive role of "extended family" in their academic development was significant. Participants shared experiences of how relatives played a proactive role in ensuring that they were emotionally and academically supported. Additionally, family members' aspirations for the participants were also shared; parents maintained high hope and ambition for their children. Although scholars (Sato \& Hodge, 2015) have considered the role of familial support for international students, family as an active and aspiring system committed to the students' professional development is an underexplored area in counseling research. Additionally, participants also indicated that peer mentorship played an important role in strengthening the international student support system. As participants experienced difficulties adjusting in a foreign country, their peer mentors offered support in the form of advice or transportation to other international students on campus. The presence of a supportive network of peer mentors is potentially indicative of leadership within the IGSC community.

Through participants' narratives, it was also clear that they expected efforts to facilitate cross-cultural transitions to be bidirectional. For instance, IGSCs wanted their education programs to understand their unique cultural practices that dictate work-life balance and values concerning workaholism. Thus, participants viewed cultural adjustment as a relational and interactive perspective as opposed to a cocooned process governed by individual international students' actions and inactions.

\section{Intrapersonal Psychosocial Strengths}

Participants in the study applied their intrapersonal psychosocial strengths such as self-reliance, strategic use of campus resources, academic persistence, and heightened critical consciousness to navigate through their study abroad experiences. These findings align with those by Yakunina et al. (2013) and other scholars (He \& Hutson, 2018; Jones et al., 2018) who noted that international students possessed various strengths, such as personal growth initiative, that had a positive effect on their acculturative stress and level of adjustment in the host culture.

Through our data analyses, it was evident that each participant developed a distinctive set of assets to support their well-being and academic pursuits. Their unique combination of strengths appeared to depend on their past experiences of living abroad, age, and particular needs. Furthermore, these strengths were not stagnant. Instead, they evolved and were responsive to the participants' changing circumstances. For instance, Fani and T. L used office hours to improve their grades, whereas A. Z. relied on campus events to distract herself from academic pressure. Their stories of perseverance are comparable to the findings by He and Hutson (2018), who noted that all their participants in their study on international students were driven to meet the program requirements. As they experienced intense academic pressure in the form of a workaholic advisor or challenging coursework, they adopted combinations of these strengths to keep themselves psychologically healthy and academically competent. Despite the critical role played by values such as perseverance and resourcefulness, counseling researchers have not explored the psychological benefits of the same for IGSCs.

Participants' awareness of their strengths evolved as they stayed in the United States. They came to recognize that although they were insulated from worldly challenges as they lived in their home countries, successfully relocating and surviving in a foreign country instilled confidence within them. According to them, another change they observed was heightened awareness of social injustices in the host country. Participants' deeper awareness of critical issues of power and privilege are comparable 
to findings by Wick and colleagues (2019), who observed that study abroad experience allowed their participants to gain a deeper understanding of embedded social injustices within the host and home country. These deeper insights resulted in participants' commitment to engage in social justice work to remove the biases. Participants in the present study learned about the nuances of social hierarchies (e.g., racism, religionism) and how they impact their everyday experiences as IGSCs.

Although the IGSCs in the present study applied a variety of strengths, we wish to iterate that the responsibility for their well-being cannot be placed solely on these participants. Far away from their families and home cultures, international students experience multiple stressors, such as visa restrictions and xenophobia (Anandavalli et al., 2020). Anandavalli and colleagues (2020) recommended multiple strategies for counselors and larger institutions to offer meaningful support to international students during uncertain sociopolitical times, such as the ongoing COVID-19 pandemic. Adopting a bioecological approach, Anandavalli and colleagues indicated that professional counselors must be equipped to support international students at various levels of the systems (e.g., culturally responsive counseling within the microsystem, political advocacy within the macrosystem). Thus, although participants in the present study exercised multiple psychosocial strengths to support their well-being, structural support and advocacy are necessary to offer them a safe and welcoming study abroad experience.

\section{Implications}

In qualitative inquiries, given a relatively smaller and more homogenous sample, transferability of findings to different settings should be based on counselors' and researchers' deep and contextualized knowledge.

\section{Counseling and Counselor Education Implications}

Firstly, participants indicated that they employed multiple strengths to meet diverse needs (e.g., cultural celebrations, academic excellence). Accordingly, counselors are advised to explore IGSCs' strengths. To achieve this, counselors can use strengths-based intake interviews and experiential activities that leverage their strengths (e.g., creative visualization of familial support). Anandavalli (2021) discussed using strengths-based counseling with international students of color, infusing an asset-based approach by using community cultural wealth theory and identifying six potential psychosocial strengths. Secondly, counselors can also collaborate with other relevant communities to offer culturally sensitive mental health support. For instance, given their reliance on religious beliefs, some IGSCs may benefit from counseling interventions that incorporate their spiritual traditions. Additionally, mental health counselors may collaborate with local religious leaders to offer culturally responsive psychoeducation on mental well-being. Thirdly, given counselors' role as advocates of change (Ratts et al., 2015), they can offer anti-implicit bias training and related anti-racism workshops for faculty and staff working with IGSCs. Furthermore, many IGSC participants in the study reported experiencing growth and heightened critical consciousness as a result of studying abroad. Safe support groups run by culturally responsive counselors can offer IGSCs a platform to process their unique journey. Given the presence of stigma against counseling among some international cultures (Pendse \& Inman, 2017), these support groups could be offered in destigmatizing spaces such as student lounges.

Within the counseling profession, the proportion of international students enrolled in master's-level and doctoral programs is increasing. Although the exact number of IGSCs within counselor education is unknown, existing literature indicates that over $4 \%$ of doctoral students in accredited counselor education programs identify as international students (Council for Accreditation of Counseling and 
Related Educational Programs, 2015). Counselor educators may model an intersectional and strengthsbased approach by appropriately identifying the strengths of IGSCs and actively incorporating them in advising and mentoring relationships.

\section{Research Implications}

The present study offers several directions for future inquiries. Firstly, within the international community, there is extensive diversity, and deeper knowledge of various intersectional groups is needed. For instance, little is known in the counseling field about the challenges that LGBTQ+ international students face and their unique strengths and support systems. Exploring the unique psychosocial assets they employ could offer much-needed perspective on the heterogeneity of international students' experiences. Secondly, at present there is no instrument to measure IGSCs' psychosocial strengths. Instrument development may aid counselors in assessing international students' strengths, applying them appropriately in the counseling setting, and measuring any changes, especially as a longitudinal study. Lastly, research on training strategies to enhance counselors' competence in working with international students is urgently needed, especially in the area of asset-based training. As international students are exposed to the effects of increasingly fragile sociopolitical periods, such as COVID-19-prompted Sinophobia, counselors are increasingly likely to be called to respond to this community's needs (Anandavalli et al., 2020). Counselor educators are invited to develop training modules to adequately prepare student counselors to work with IGSCs in a strengths-based approach.

\section{Limitations}

Although valuable data were gained through this inquiry, the study is not without its limitations. Interviews for the current study were completed in English. Given that for many international students English is not their preferred language, data and the narratives offered could have been different if alternative languages for interviewing had been offered. Also, all the participants in the study were located in the American South. Potentially, narratives could have differed if participants were from states with larger international student populations (e.g., New York, California). Furthermore, although unintentional, both the researchers and participants in this study identified as cisgender female. Inquiries involving all genders' experiences as IGSCs could offer varied and salient details on their narratives.

\section{Conclusion}

Counselors must have knowledge of IGSCs' psychosocial strengths to leverage them effectively in counseling. Researchers have repeatedly indicated that incorporating minoritized clients' strengths in counseling results in improved resilience, confidence, and self-efficacy. However, despite persistent calls by scholars, extant literature offers limited information on IGSCs' strengths. Our IPA investigation with eight IGSC participants provides a rich description of unique psychosocial strengths they employ to support their mental health. Professional counselors can expand their cultural competence to work with this community by examining and implementing these findings.

\section{Conflict of Interest and Funding Disclosure}

The authors reported no conflict of interest or funding contributions for the development of this manuscript. 
The Professional Counselor I Volume 11, Issue 2

\section{References}

Anandavalli, S. (2021). Strengths-based counseling with international students of color: A community cultural wealth approach. Journal of Asia Pacific Counseling, 11(1), 111-124. https://doi.org/10.18401/2021.11.1.7

Anandavalli, S., Harrichand, J. J. S., \& Litam, S. D. A. (2020). Counseling international students in times of uncertainty: A critical feminist and bioecological approach. The Professional Counselor, 10(3), 365-375. https://doi.org/10.15241/sa.10.3.365

Chirikov, I., \& Soria, K. M. (2020). International students' experiences and concerns during the pandemic. SERU Consortium, University of California - Berkeley and University of Minnesota. https://docs.google.com/ document/d/11Zdc4dWiX9C 8o1vH-4H7XokIHoJ4yijVNRv0vS3tBE/edit\#heading=h.ivkygr983tef

Click, A. B. (2017). Stress, adaptation, growth: International students' approaches to scholarly research and academic integrity. Proceedings of the Association for Information Science and Technology, 54(1), 642-644. https://doi.org/10.1002/pra2.2017.14505401099

Council for Accreditation of Counseling and Related Educational Programs. (2015). CACREP vital statistics 2015: Results from a national survey of accredited programs. http://www.cacrep.org/wp-content/uploads/2019/05/ 2015-CACREP-Vital-Statistics-Report.pdf

Craig, S. L. (2013). Affirmative Supportive Safe and Empowering Talk (ASSET): Leveraging the strengths and resiliencies of sexual minority youth in school-based groups. Journal of LGBTQ Issues in Counseling, 7(4), 372-386. https://doi.org/10.1080/15538605.2013.839342

Craig, S. L., \& Furman, E. (2018). Do marginalized youth experience strengths in strengths-based interventions? Unpacking program acceptability through two interventions for sexual and gender minority youth. Journal of Social Service Research, 44(2), 168-179. https://doi.org/10.1080/01488376.2018.1436631

Crenshaw, K. W. (1989). Demarginalizing the intersection of race and sex: A Black feminist critique of antidiscrimination doctrine, feminist theory and antiracist politics. University of Chicago Legal Forum, 1989(1), 139-167. https://chicagounbound.uchicago.edu/cgi/viewcontent.cgi?article=1052\&context=uclf

Day-Vines, N. L., \& Terriquez, V. (2008). A strengths-based approach to promoting prosocial behavior among African American and Latino students. Professional School Counseling, 12(2), 170-175. https://doi.org/10.1177/2156759X0801200204

Dovchin, S. (2020). The psychological damages of linguistic racism and international students in Australia. International Journal of Bilingual Education and Bilingualism, 23(7), 804-818. https://doi.org/10.1080/13670050.2020.1759504

Erichsen, E. A., \& Bolliger, D. U. (2011). Towards understanding international graduate student isolation in traditional and online environments. Educational Technology Research and Development, 59(3), 309-326. https://doi.org/10.1007/s11423-010-9161-6

George Mwangi, C. A., Changamire, N., \& Mosselson, J. (2019). An intersectional understanding of African international graduate students' experiences in U.S. higher education. Journal of Diversity in Higher Education, 12(1), 52-64. https://doi.org/10.1037/dhe0000076

Girmay, M. (2019). Understanding the mental and physical health needs and acculturation processes of international graduate students in the United States. Journal of Comparative $\mathcal{E}$ International Higher Education, 11(Fall), 10-17. https://doi.org/10.32674/jcihe.v11iFall.1071

Guba, E. G. (1981). Criteria for assessing the trustworthiness of naturalistic inquiries. Educational Communication and Technology, 29(2), 75-91.http://www.jstor.org/stable/30219811

Harry, B., Klingner, J. K., \& Hart, J. (2005). African American families under fire: Ethnographic views of family strengths. Remedial and Special Education, 26(2), 101-112. https://doi.org/10.1177/07419325050260020501

He, Y., \& Hutson, B. (2018). Exploring and leveraging Chinese international students' strengths for success. Journal of International Students, 8(1), 87-108. https://doi.org/10.5281/zenodo.1101037

Jones, L., O'Connor, E., \& Boag-Hodgson, C. (2018). International psychology students use multiple strengths to enhance their learning and performance on work placements. Australian Psychologist, 53(6), 505-516. https://doi.org/10.1111/ap.12351

Lee, J. J., \& Rice, C. (2007). Welcome to America? International student perceptions of discrimination. Higher Education, 53(3), 381-409. https://doi.org/10.1007/s10734-005-4508-3 
Lemieux, M., Colazo, J. M., Kienka, T., \& Zhakyp, A. (2020). A basis to be here: Stories from international graduate students in the United States. Cell Reports Medicine, 1(6), 1-4. https://doi.org/10.1016/j.xcrm.2020.100100

Lin, S.-Y., \& Scherz, S. D. (2014). Challenges facing Asian international graduate students in the US: Pedagogical considerations in higher education. Journal of International Students, 4(1), 16-33. https://doi.org/10.32674/jis.v4i1.494

Moores, L., \& Popadiuk, N. (2011). Positive aspects of international student transitions: A qualitative inquiry. Journal of College Student Development, 52(3), 291-306. https://doi.org/10.1353/csd.2011.0040

Mori, S. C. (2000). Addressing the mental health concerns of international students. Journal of Counseling $\mathcal{E}$ Development, 78(2), 137-144. https://doi.org/10.1002/j.1556-6676.2000.tb02571.x

National Association of Foreign Student Advisers. (2020). Losing talent 2020: An economic and foreign policy risk America can't ignore. https://www.nafsa.org/sites/default/files/media/document/nafsa-losing-talent.pdf

National Board for Certified Counselors. (2016). National Board for Certified Counselors (NBCC) code of ethics. https://www.nbcc.org/Assets/Ethics/NBCCCodeofEthics.pdf

Pendse, A., \& Inman, A. G. (2017). International student-focused counseling research: A 34-year content analysis. Counselling Psychology Quarterly, 30(1), 20-47. https://doi.org/10.1080/09515070.2015.1128395

Pottie-Sherman, Y. (2018). Retaining international students in northeast Ohio: Opportunities and challenges in the 'age of Trump.' Geoforum, 96, 32-40. https://doi.org/10.1016/j.geoforum.2018.07.015

Ratts, M. J., Singh, A. A., Nassar-McMillan, S., Butler, S. K., \& McCullough, J. R. (2015). Multicultural and social justice counseling competencies. https://www.counseling.org/docs/default-source/competencies/multiculturaland-social-justice-counseling-competencies.pdf?sfvrsn=20

Sato, T., \& Hodge, S. R. (2015). Japanese exchange students' academic and social struggles at an American University. Journal of International Students, 5(3), 208-227. https://doi.org/10.32674/jis.v5i3.417

Smith, J. A. (1996). Beyond the divide between cognition and discourse: Using interpretative phenomenological analysis in health psychology. Psychology $\mathcal{E}$ Health, 11(2), 261-271. https://doi.org/10.1080/08870449608400256

Smith, J. A., \& Osborn, M. (2007). Interpretive phenomenological analysis. In J. A. Smith (Ed.), Qualitative psychology: A practical guide to research methods (2nd ed., pp. 53-80). SAGE.

Straker, J. (2016). International student participation in higher education: Changing the focus from "international students" to "participation." Journal of Studies in International Education, 20(4), 299-318. https://doi.org/10.1177/1028315316628992

Tomlinson-Clarke, S. M., \& Georges, C. M. (2014). DSM-5: A commentary on integrating multicultural and strength-based considerations into counseling training and practice. The Professional Counselor, 4(3), $272-281$. https://doi.org/10.15241/stc.4.3.272

Toporek, R. L., \& Cohen, R. F. (2017). Strength-based narrative résumé counseling: Constructing positive career identities from difficult employment histories. The Career Development Quarterly, 65(3), 222-236. https://doi.org/10.1002/cdq.12094

U.S. Immigration and Customs Enforcement. (2020, July 6). SEVP modifies temporary exemptions for nonimmigrant students taking online courses during fall 2020 semester. https://www.ice.gov/news/releases/sevp-modifiestemporary-exemptions-nonimmigrant-students-taking-online-courses-during

Wang, Q., \& Hannes, K. (2014). Academic and socio-cultural adjustment among Asian international students in the Flemish community of Belgium: A photovoice project. International Journal of Intercultural Relations, 39, 66-81. https://doi.org/10.1016/j.ijintrel.2013.09.013

Wick, D., Willis, T. Y., Rivera, J., Lueker, E., \& Hernandez, M. (2019). Assets-based learning abroad: First-generation Latinx college students leveraging and increasing community cultural wealth in Costa Rica. Frontiers: The Interdisciplinary Journal of Study Abroad, 31(2), 63-85. https://doi.org/10.36366/frontiers.v31i2.455

Yakunina, E. S., Weigold, I. K., Weigold, A., Hercegovac, S., \& Elsayed, N. (2013). International students' personal and multicultural strengths: Reducing acculturative stress and promoting adjustment. Journal of Counseling \& Development, 91(2), 216-223. https://doi.org/10.1002/j.1556-6676.2013.00088.x

Yao, C. W. (2018). “They don't care about you": First-year Chinese international students' experiences with neoracism and othering on a U.S. campus. Journal of the First-Year Experience E Students in Transition, 30(1), 87-101. 
Yao, C. W., Briscoe, K. L., \& Rutt, J. N. (2020). In the aftermath of a racialized incident: Exploring international students of color's perceptions of campus racial climate. Journal of Diversity in Higher Education. Advance online publication. https://doi.org/10.1037/dhe0000179

Yosso, T. J. (2005). Whose culture has capital? A critical race theory discussion of community cultural wealth. Race Ethnicity and Education, 8(1), 69-91. https://doi.org/10.1080/1361332052000341006 